\title{
Modeling and Simulation of Multiple-Input Converter System with Equally Drawn Source Power
}

\author{
Taufik, Brian Butterfield \\ Electrical Engineering Department \\ Cal Poly State University \\ San Luis Obispo, CA 93407 USA \\ taufik@calpoly.edu
}

\author{
Makbul Anwari \\ Faculty of Electrical Engineering \\ Universiti Teknologi Malaysia \\ 81310 UTM Skudai, Malaysia \\ makbul@ieee.org
}

\author{
Mohammad Taufik \\ Department of Physics \\ Universitas Padjadjaran \\ Bandung 40132, Indonesia \\ mhtaufik@yahoo.com
}

\begin{abstract}
This paper presents the modeling of a proposed multiple input, single output converter system for use mainly to interface several renewable energy sources into a single load connection point. More specifically, modeling of a system involving ac and de supplied to the multiple inputs, single output DC-DC converter will be described and then verified via simulation using OrCAD Pspice to exhibit its functionality. Results of the simulation of one model show the achievement of equally supplied source currents. Details of the modeling and the accompanying computer simulation of the proposed converter will be discussed.
\end{abstract}

Keywords-Power Converter, Renewable Energy Sources

\section{INTRODUCTION}

Traditionally, power-processing circuits that are lineartechnology based tend to perform far from ideal [1]. An efficiency characterization of a typical audio amplifier, for example, may offer efficiencies of less than $50 \%$ [2]. This is mainly because linear power processor such as linear regulators employs a transistor operating continuously in its linear region in its main power channeling device. This results in a significant amount of power loss at the transistor. Despite the serious drawback, linear power processing technique offers market desirable attributes, namely simplicity and reliability. The technique is well known for its stability, noise-free, and exceptional line and load regulations. Its simplicity in design also brings their system costs attractively low [3]. However, today's applications demand more on highly efficient, hence more compact, converters. This begs for an alternative technique for processing power.

Today, the ability to obtain highly efficient power converters is achieved through power electronic technology. In particular, a transistor in power electronics is operated as a switch, i.e. saturation region for turn on and cutoff region for turn off to achieve the desired output parameters. This further lessens the loss of the transistor, and recent developments of converter utilizing transistor as a switch have been able to push converter's efficiencies approaching 100\% [4-6]. Yet another benefit of power electronic converters is their high switching frequency operation, ranging from kilo Hertz to Mega Hertz. This further implies their inherent reduction of weight and space requirements, as compared to those of linear-technology based circuits. The higher efficiency and less bulky attributes of power electronic circuits which make them the most attractive technique in today's power processing circuits.

The higher switching frequency operation of power electronic converters unfortunately brings about a major challenge in their design. This is mainly due to the detrimental effects of the stray components which worsen at high frequency operation and are difficult to predict. Hence, it is a common practice in industry to supplement converter design with modeling and simulation of the converter to ensure the desired operation of the converter. The proposed multi-input single-output converter system presented in this paper is of no exception. Before hardware prototyping and due to the complexity of the system, modeling and then simulation of the proposed converter will have to be conducted.

\section{MultiPle-InPUt Single-OUtPut CONVERTER}

Solar photovoltaic (PV) systems typically contain one or more solar panels as a primary DC source, a battery bank for energy storage, electronics for power flow control, and may or may not provide the ability to connect to an ac grid for bidirectional power flow. In this paper, a proposed converter system that interfaces simultaneously the ac and dc sources (battery) to a varying load will be presented. The main objective of the converter system is to allow power to be equally drawn from both sources such that higher efficiency of overall PV's electrical system could be achieved and energy cost for predetermined time during the day could be minimized.

The proposed converter system is unique since power electronic converters typically have only one input and may have one or more outputs. This further emphasizes the importance of modeling the proposed due to lack of prior study or design of such converter system. The modeling and then the simulation of the proposed converter system was done using OrCAD PSpice due to its popularity as the modeling and simulation tool in industry for circuit analysis [7]. 


\section{REQUIREMENTS}

The primary concern and design requirement for the proposed converter lies on the control of the time-varying inputs to equally transfer power to a time-varying load. Another important design requirement is the over-current protection to prevent damage to any of the input source devices. The basic block diagram of the system is illustrated in Figure 1.

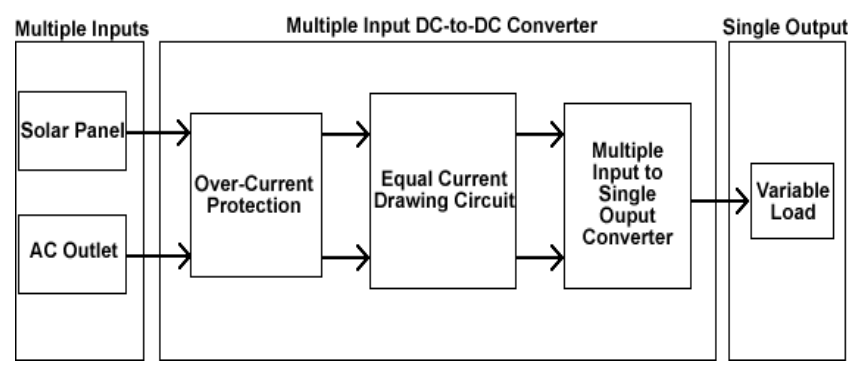

Figure 1. Basic block diagram showing project requirements

As a major step after component selection is completed, modeling and simulation of the proposed converter follows to verify whether desired parameters and performance are satisfied.

To achieve the equal sharing of the input current, current mirroring method using Darlington transistors is employed whose model is shown in Figure 2. Because the input base currents and base-emitter voltages are the same for both transistors, the non-referenced side of the current mirror should mimic the current amplification of the referenced side. The dotted box shows the circuitry that is internal to the Darlington transistor. As indicated in Figure 2, the Darlington current mirror serves as the main function that draws the equal currents from the sources. The Buck converters help keep the voltage at the collector terminals at roughly the same potential which should assist in the accuracy of the current mirror.

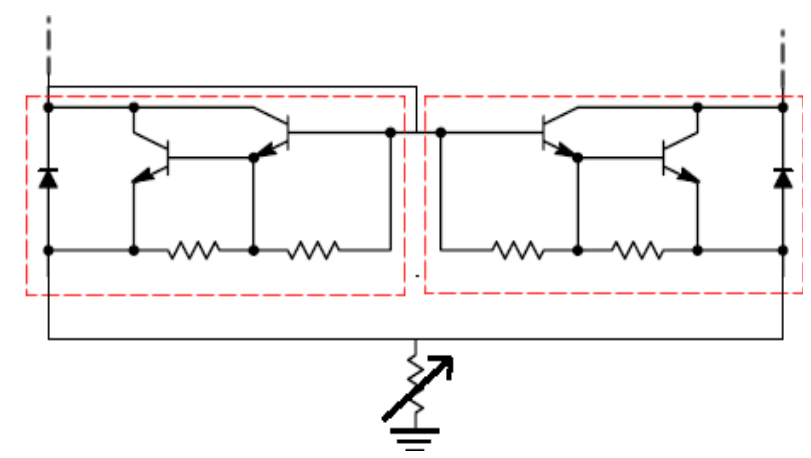

Figure 2. Darlington current mirror circuit model

\section{CIRCUIT MODELS}

Figure 3 depicts a model of the proposed converter system using OrCAD Pspice. As shown, the proposed system consists of two step-down or Buck regulators. The switches are modeled using a component called Sbreak which mimics an ideal switch in terms of its transition property, but may still possess conduction loss should the user choose to. The model also represents a closed loop system which is required to enable both step-down regulators to maintain their output voltages. The controller in each regulator employs a model available in Pspice regulator library rather than creating its model from discrete components. The controller chosen is the SG1843 model which is a widely used controller for DC-DC converters. The solar panel is modeled as a 24 volt DC voltage source, whereas the AC input is modeled with an ideal sinusoidal voltage source. As illustrated in Figure 3, the top Buck regulator which receives power from the solar panel provides a power path to one collector of the current mirror for the output stage. The bottom Buck regulator provides a power path for the $\mathrm{AC}$ power, first through a rectifying diode bridge, and then through the actual converter to the other collector of the current mirror.

The load in the model is represented by a variable resistor. In actual implementation of the converter system, the load will actually be drawn from an electronic load which acts just like a current source. Since the output voltage of the converter is tightly maintained at a certain level, the load model using adjustable resistor should suffice.

Another model of the proposed converter system is shown in Figure 4. The figure illustrates a two dimensional view of the proposed circuit to emphasize the provision and existence of the parallel paths in the system. This model was a more realistic model than the one previously described since the entire Buck regulator is represented by a real world model of a Buck regulator chip instead of discrete components.

\section{SimUlation RESUlts}

Figure 4 shows that the proposed circuit model is fully functional, having both $\mathrm{DC}$ (from solar panels) and $\mathrm{AC}$ (from wall outlet) inputs, satisfying desired equal-source current requirement at full load, where the load was set to draw approximately 20 A. Figure 4 further shows that the average switch current at approximately 5 A each corresponding to the $50 \%$ duty cycle operation of each Buck regulator. This validates the occurrence of equal current or power sharing into both Buck regulators. The desired equal current characteristic was also simulated and verified at $10 \%$ load as shown in Figure 5. 


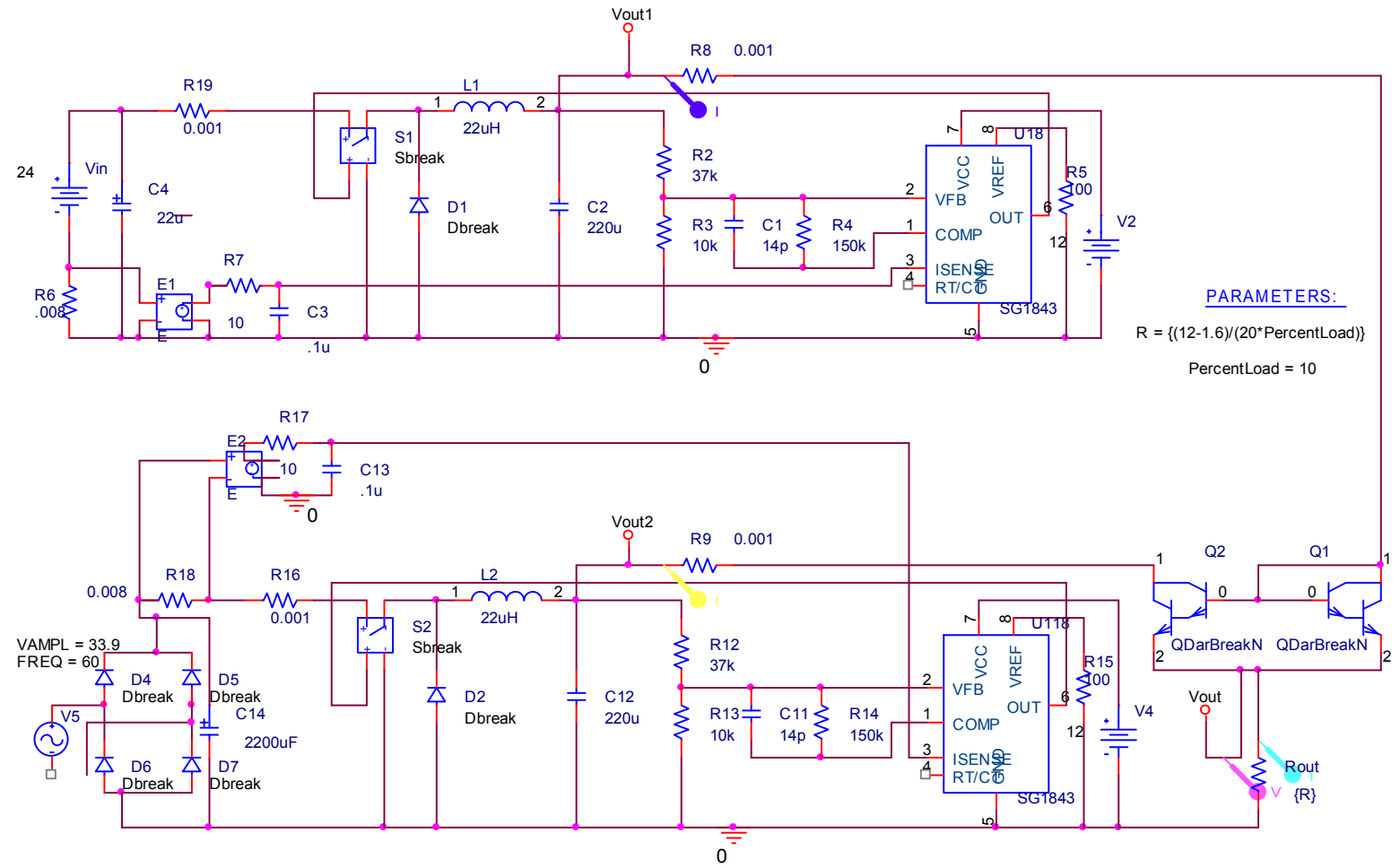

Figure 3. Pspice circuit model of the proposed converter system

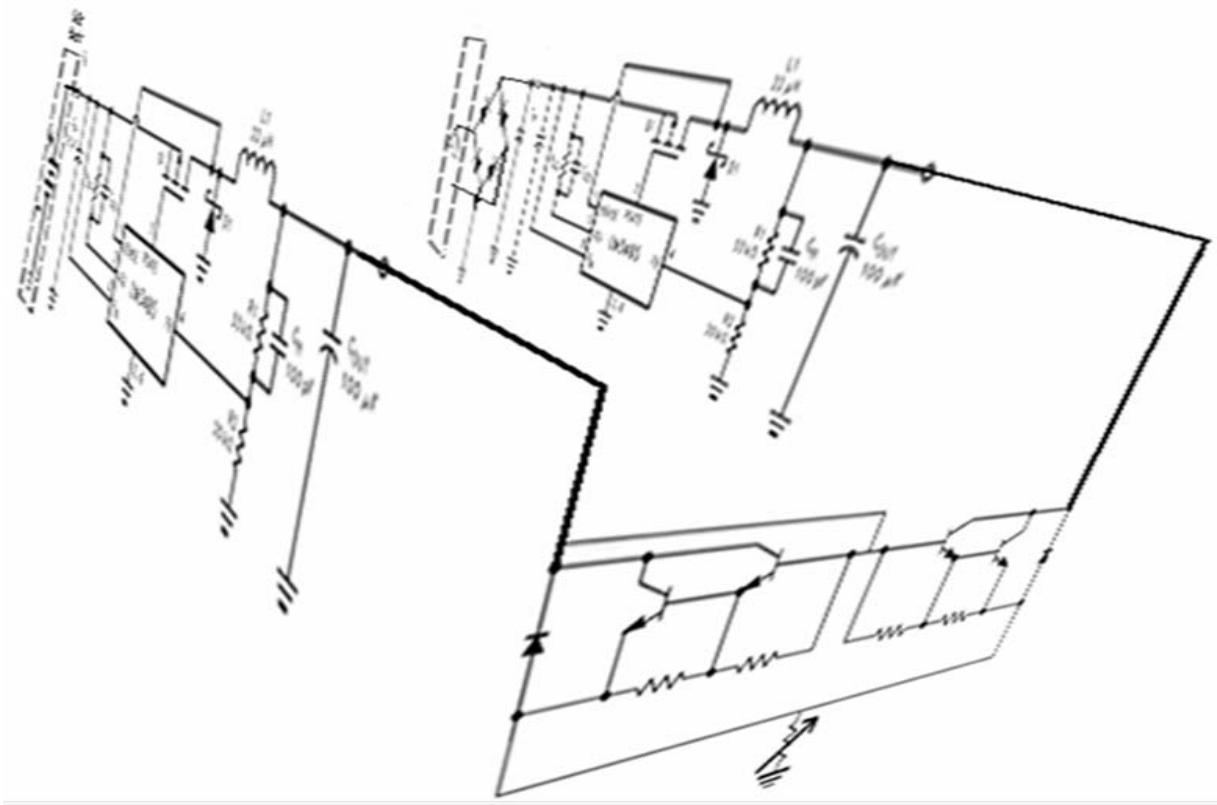

Figure 4. A More realistic circuit model of the proposed converter system 


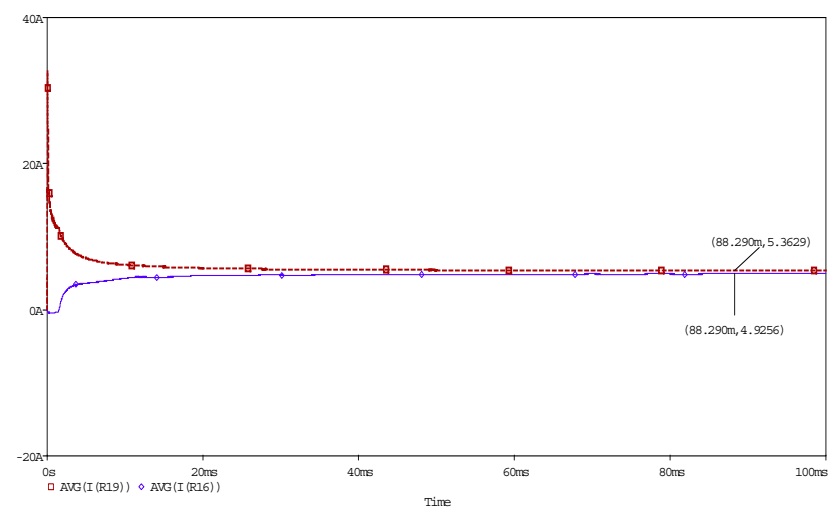

Figure 5. Simulation result at full load

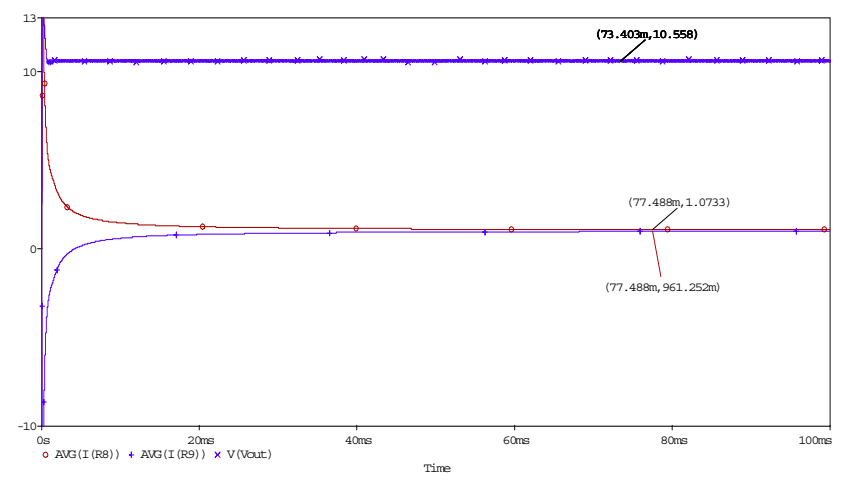

Figure 6. Simulation result at light load

Comparison of the two figures (Figures 4 and 5) indicates that the equal current sharing characteristic is better at the lighter load. This is true not only at $10 \%$ load but also at other lighter loads in general. This is due to at least a couple of reasons. First, several components used in the model incorporate losses. This includes the switches (Sbreak), diodes (Dbreak), and Darlington transistors (QDarBreakN). This would result in larger forward conduction losses at higher output current following the Ohm's Law. Hence the output voltage (similarly, the output current) of each Buck regulator will be closer to the desired value at lighter loads than it is at heavier loads. Another reason for the slight difference in the equal sharing performance at different loads is by the lack of optimum feedback compensation in each controller of the Buck regulator. In the circuit models shown previously, the primary objective of the modeling was to verify whether the proposed converter system could achieve the equal source current, with little priority of getting the most optimum performance of the system optimum. As such, the feedback compensation incorporated in the models was only set to obtain stable outputs.

\section{CONCLUSION}

In this paper, the modeling of a proposed converter system that interfaces multiple inputs has been presented. The proposed converter system is one approach that models a load that is fed by multiple energy sources such as those found in residential, commercial or industrial facilities incorporating renewable energy sources. Although the model described in this paper utilizes solar panels and ac source from the utility line, its use may be extended to other systems with different types of energy sources such as fuel cells, wind turbines, etc.

The proposed converter system employs buck regulators whose objective is to ensure equal sharing of current drawn from each source. Due to the complexity of the proposed system, modeling is therefore necessary. Two models were developed as discussed in this paper. Results from one of the models show that the equal sharing of current was indeed achieved. Although not included in this paper, these results were later compared with actual hardware implementation and observed to show good agreement with the hardware results.

\section{REFERENCES}

[1] Pressman, A., and Billings, K., Switching Power Supply Design, McGraw-Hill, 2009.

[2] Krishnamurthy, K.; Vetury, R.; Keller, S.; Mishra, U.; Rodwell, M.J.W.; Long, S.I., "Broadband GaAs MESFET and GaN HEMT Resistive Feedback Power Amplifiers", IEEE Journal of SolidState Circuits, Vol. 35, Sept. 2000.

[3] Lenk, R., Practical Design of Power Supplies, Wiley and IEEE Press, 2005.

[4] Fatemi, N.S.; Hoffman, R.W.; Lowe, R.A.; Jenkins, P.P.; Garverick, L.M.; Wilt, D.M.; Scheiman, D., "High Efficiency Converters for Thermophotovoltaic Applications", Proceedings of Energy Conversion Engineering Conference, 1996.

[5] Byung Duk Min,; JongPil. Lee,; Jong-Hyun Kim,; Tae-Jin Kim,; Dong-Wook Yoo,; Kang Ryoul Ryu,; Jung Joong Kim,; Eui-Ho Song,; "A Novel Grid-Connected PV PCS with New High Efficiency Converter", Proceedings of International Conference on Power Electronics, 2007.

[6] Jitaru, I.D.; "High Efficiency Converter Using Current Shaping \& Synchronous Rectification", Proceedings of Telecommunications Energy Conference, 2002.

[7] Mitzner, K., Complete PCB Design Using OrCad Capture and Layout, Newness, 2007. 